\title{
Einfluss der Physikochemie auf die Abtrennung nanoskali- ger Partikel aus Flüssigkeiten
}

\author{
Hermann Nirschl*
}

\begin{abstract}
Der folgende Beitrag hat zum Ziel, die verfahrenstechnischen Grundlagen über den Einfluss der Physikochemie auf die Abtrennung feinster, nanoskaliger Partikel aus einer Suspension zu ordnen und zusammenzufassen. Im Mittelpunkt stehen dabei die in den mechanischen Trenntechniken bedeutsamsten Trennprinzipien, die Sedimentation und die kuchenbildende Filtration. Ausgehend von den physikochemischen und verfahrenstechnischen Grundlagen im Bereich der Sedimentation und Filtration versucht der Beitrag, die Einflussgrößen auf die Abscheidung zu identifizieren und die wesentlichen, experimentell ermittelten Zusammenhänge darzustellen.
\end{abstract}

Schlagwörter: Filtration, Nanopartikel, Sedimentation, Suspension

Eingegangen: 31. Juli 2007; akzeptiert: 03. August 2007

\section{Einleitung}

Die Separation von feinsten, oftmals auch nanoskaligen Partikeln wird in jüngster Vergangenheit in vielen Prozessen gefordert. Durch die rasante Entwicklung in der Herstellung und Anwendung nanoskaliger Partikeln müssen diese zum einen während oder auch nach dem Herstellungsprozess aus der gasförmigen oder flüssigen Umgebung abgetrennt werden. Zum anderen bereitet die Separation in Form einer Klassierung oder Abscheidung während eines Verarbeitungsprozesses erhebliche Probleme. In erster Linie liegt dies natürlich an dem bei immer kleiner werdenden Partikelgrößen verschwindenden Einfluss der Massenkräfte. Stattdessen treten die durch die Oberflächenvergrößerung mit abnehmender Partikelgröße immer stärker dominierenden Grenzflächenkräfte in den Vordergrund. Obwohl derzeit an vielen Forschungsstellen an der Synthese oder der Verarbeitung von feinsten, nanoskaligen Partikelsystemen intensiv gearbeitet wird, bleiben doch noch viele grundlegende Fragestellungen aus der Verfahrenstechnik unverstanden.

\section{Physikochemische Grundlagen}

Neben den dispersen Eigenschaften bestimmen interpartikuläre Kräfte die Struktur und die mechanischen Eigenschaften feindisperser Suspensionen. Die interpartikulären Gesamtwechselwirkungen hängen dabei stark von den physikochemischen Parametern wie $\mathrm{pH}$-Wert und Ionenstärke ab. Van-der-Waals (vdW)-Kräfte zwischen Partikeln des gleichen Stoffes in wässrigen Suspension sind stets anziehend [1]. Die physikalische Ursache beruht dabei auf der Wechselwirkung von Dipolmomenten von Atomen und Molekülen. Es kommen sowohl permanente als auch fluktuierende Dipole vor, die durch gegenseitige Beeinflussung der Elektronen benachbarter Atome hervorgerufen werden [2]. Hamaker [3] sowie Verwey und Overbeek [4] geben eine Gleichung für das Wechselwirkungspotenzial $V_{\mathrm{a}}$ zweier kugelförmiger Partikel mit Abstand $H$ an. Für kleine Abstände $H$ existiert folgende Näherung [5]:

$V_{\mathrm{a}}=-\frac{A}{12}\left(\frac{L}{H}+2 \ln \frac{H}{L}\right)$

mit $L$ als einer geometrischen Konstanten.

Die maßgebliche Stoffkonstante, die bei gegebener Geometrie allein die Höhe der vdWWechselwirkung bestimmt, ist die HamakerKonstante $A$. In erster Näherung ist $A$ vom Elektrolytgehalt der Suspension und vom Partikelabstand unabhängig. Eine steigende Ionenstärke hat in der Realität, ebenso wie ausgeprägte Adsorptionsschichten auf der Partikeloberfläche, ebenfalls einen absenkenden Einfluss auf die vdW-Anziehung [4].
Neben den dispersen Eigenschaften bestimmen interpartikuläre Kräfte die Struktur und die mechanischen Eigenschaften feindisperser Suspensionen, die stark von $\mathrm{pH}$ Wert und lonenstärke abhängen. 
Die bedeutsamsten repulsiven Wechselwirkungen in wässrigen Fest/Flüssig-Systemen entstehen aufgrund elektrostatischer Abstoßungskräfte zwischen gleichnamig geladenen Partikeloberflächen. Als Ursachen für Oberflächenladungen kommen die Protolyse, eine isomorphe Substitution und eine spezifische Adsorption von Ionen in Frage. Bei der Protolyse, die insbesondere bei oxidischen Werkstoffen zu finden ist, entstehen Oberflächenladungen durch Adsorption bzw. Desorption von Protonen an Hydroxyl- oder Sauerstoffionen im Oberflächenbereich. Die Ladungsdichte ist somit vom $\mathrm{pH}$ der Suspension abhängig. Eine isomorphe Substitution tritt vor allem bei Tonmineralien, wie Schichtsilikaten, auf [1]. Neben der bereits für Oxide beschriebenen Protolyse von Protonen können sich auch andere Ionen spezifisch an der Partikeloberfläche anlagern und so die Oberflächenladung beeinflussen [6].

Es existieren zahlreiche Modelle unterschiedlicher Komplexität zur Beschreibung der Ionenatmosphäre und des Potenzialverlaufs. Eine Übersicht findet sich in [1]. An die Partikeloberfläche schließt sich eine starre Schicht aus adsorbierten Ionen an, die als Sternschicht bezeichnet wird. Darin werden spezifisch adsorbierte Ionen näher an die Partikeloberfläche herangerückt und bilden die sog. innere Helmholtz-Schicht, während nicht-spezifisch d.h. elektrostatisch adsorbierte Ionen die äußere Helmholtz-Schicht bilden. Der Potenzialverlauf in der inneren und äußeren Helmholtz-Schicht ist linear. Dieses Modell lässt sich noch ausbauen, indem weitere Adsorptionsebenen eingefügt werden, die z.B. die Wertigkeit oder die Hydratation der Ionen berücksichtigen.

An die Sternschicht schließt sich die sog. diffuse Schicht an, in der das Potenzial exponentiell abklingt. Die thermische und die elektrostatische Energie stehen im dynamischen Gleichgewicht, das den Konzentrationsgradienten aufrechterhält. Eine exakte Lösung der den Potenzialverlauf zugrundeliegenden Poisson-Boltzmann Gleichung für realitätsnahe Bedingungen, d. h. mehrwertige Ionen in Doppelschichten um harte, kugelförmige Partikel, ist mathematisch sehr aufwändig [1, 7]. Die Debye-Länge $L_{\mathrm{D}}$ ist die charakteristische Abklinglänge des Potenzials, d.h. in der Entfernung $L_{\mathrm{D}}$ in Feldrichtung von einem beliebigen Punkt in der diffusen Schicht sinkt das Potenzial auf den e-ten Teil (36\%) ab.

Unter Verwendung des Zetapotenzials $\zeta$ geben Hogg et al. [7] folgenden Ausdruck zur Berechnung der elektrostatischen Kraft zwischen zwei Partikeln gleicher Größe an:

$F_{\mathrm{r}}=\frac{\pi x_{\mathrm{p}} \varepsilon_{0} \varepsilon_{\mathrm{r}} \kappa \varsigma^{2} \exp (-\kappa H)}{1+\exp (-\kappa H)}$
Bei direktem Partikelkontakt treten aufgrund der gegenseitigen Durchdringung der Elektronenhüllen noch sog. Born'sche Abstoßungskräfte auf. Sie bewirken ein tief gelegenes primäres Minimum in der Gesamtwechselwirkungskurve, das jedoch für die Stabilität von Suspensionen und die Redispergierung von Partikeln kaum Bedeutung hat [1].

Das Gesamtwechselwirkungspotenzial zwischen Partikeln kann mittels der nach ihren Autoren Derjaguin und Landau [8] sowie Verwey und Overbeek [4] benannten DLVO-Theorie beschrieben werden. Unter der Annahme, dass keine gegenseitige Beeinflussung stattfindet, werden dabei die attraktiven und repulsiven Einzelpotenziale $V_{\mathrm{a}}$ und $V_{\mathrm{r}}$ additiv überlagert. Die im jeweiligen Partikelabstand wirksame Kraft ergibt sich dann als Gradient der Gesamtpotenzialkurve.

Da das elektrostatische Potenzial stark von der Ionenstärke und der wiederum pH-abhängigen Oberflächenladung bzw. dem Sternpotenzial abhängt, kann durch Änderung von $\mathrm{pH}$ und Ionenstärke die Gesamtwechselwirkungskurve beeinflusst und das Agglomerationsverhalten der Partikel verändert werden. Sinkt das Potenzialmaximum auf Werte von $10-15 \mathrm{kT} \mathrm{ab}$, reicht die thermische Energie der Teilchen aus, um es zu überwinden; es kommt zur Koagulation bzw. zur Bildung von strukturierten Agglomeraten.

Der Definition des Zetapotenzials $\zeta$ liegt die Annahme einer starren und einer diffusen Ionenschicht zugrunde, wobei letztere während der Messung bis $\mathrm{zu}$ einer bestimmten Ebene abgeschert wird. Das Partikel und der nicht abgescherte Teil der es umgebenden Ionenschicht weisen eine Ladung auf, die in der Scherebene das Zetapotenzial bewirkt. Es liefert somit einen Anhaltswert bezüglich des effektiven Oberflächenpotenzials und wird daher oft mit dem Sternpotenzial gleichgesetzt. Eine vollständige Abscherung der diffusen Schicht wird angenommen, obwohl die exakte Lage der Scherebene unbekannt ist.

Die effektive Ladung der Partikel eines Fest/ Flüssig-Systems, charakterisiert durch das Zetapotenzial, ist für dessen Eigenschaften (Agglomerationszustand, Sedimentationsverhalten, Mikrostruktur...) von ausschlaggebender Bedeutung [9]. Haupteinflussparameter sind der $\mathrm{pH}$-Wert und der Elektrolytgehalt der Suspension. Mit steigendem $\mathrm{pH}$-Wert nimmt die zunächst positive Ladung der Partikeloberfläche ab, erreicht den isoelektrischen Punkt und wird schließlich umgeladen. Je höher der Betrag des Zetapotenzials, desto größer ist die elektrostatische Abstoßung zwischen den Partikeln. Die Zugabe nicht-spezifisch adsorbierender Ionen führt zu einem steileren Abklin- 
gen des Potenzialverlaufs um das Partikel bzw. einer Kompression der diffusen Ionenschicht. Sofern eine unveränderte Lage der Scherebene vorausgesetzt wird, sinkt das Zetapotenzial ab.

Das Zetapotenzial erlaubt für praktische Zwecke häufig eine ausreichende Vorhersage des Wechselwirkungszustandes und der Stabilität einer Suspension. Es kann jedoch streng genommen nicht als alleiniger Stabilitätsindikator herangezogen werden, da die elektrostatische Wechselwirkungskraft nicht vom Absolutwert des Potenzials, sondern vom Gradienten der Potenzial-Abstandskurve abhängt [10]. Daher existieren zahlreiche Stoffsysteme, deren Verhalten nicht vollständig mit Hilfe der DLVO-Thoerie und des Zetapotenzials beschrieben werden kann, weil z. B. Hydratationskräfte dominieren.

\section{Physikochemische Einflüsse auf die Sedimentation}

Die Differenzbewegung von partikulären Feststoffen gegenüber einer Flüssigkeit, hervorgerufen von einer positiven Dichtedifferenz zwischen Feststoff und Flüssigkeit, wird als Sedimentation bezeichnet. In diesem Beitrag wird ausschließlich die Sedimentation eines Feststoffs in einer wässrigen Phase betrachtet. Je feiner die Partikel werden, desto mehr verlieren die massenbedingten Effekte an Einfluss, da sich das Verhältnis aus Oberfläche und Volumen mit abnehmender Partikelgröße vergrößert. Das Verständnis des Sedimentationsverhaltens feinster Partikel setzt daher eine Einbeziehung der durch Grenzflächeneffekte dominierten physikochemischen Phänomene zur Erklärung voraus.

Das Sedimentationsverhalten von Feststoffen ist neben den Stoffeigenschaften von Feststoff und Flüssigkeit von der Konzentration des Feststoffes abhängig. Der einfachste Ansatz zur Beschreibung der Sedimentation eines einzelnen Partikels, in theoretisch unendlicher Verdünnung, stammt von Stokes [11]. Durch eine Bilanzierung der auf ein Partikel wirkenden Kräfte, der Gewichts-, Auftriebsund Widerstandskraft, ergibt sich mittels einer einfachen Umrechnung die Sinkgeschwindigkeit eines Partikels:

$u_{\mathrm{St}}=\frac{\left(\rho_{\mathrm{p}}-\rho_{1}\right) g x_{\mathrm{p}}^{2}}{18 \eta}$

Die Sinkgeschwindigkeit einer Kugel ist damit proportional zur Dichtedifferenz zwischen Feststoff und Flüssigkeit und zum Quadrat des Durchmessers.

In praktischen Anwendungen ist die Feststoffkonzentration jedoch so hoch, dass sich

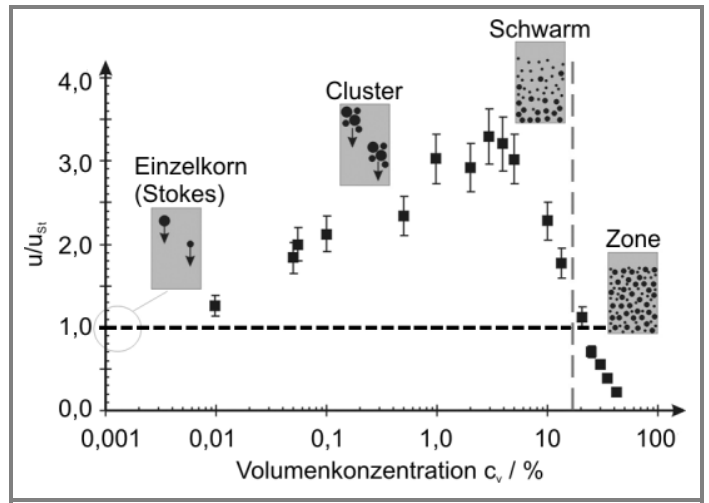

Abbildung 1. Einfluss der Feststoffkonzentration auf die Sinkgeschwindigkeit monodisperser Partikelsysteme (nach Bickert [12]).

die Partikel gegenseitig während der Sedimentation beeinflussen. An dieser Stelle gilt es auch zwischen monodispersen und polydispersen Partikelsystemen zu unterscheiden. Im Bereich sehr kleiner Feststoffkonzentrationen bilden sich gemeinsam sedimentierende Komplexe oder Cluster. Die Stromlinien der Einzelpartikel im Cluster überlagern sich, wodurch es zu einer Reduzierung des Gesamt-Strömungswiderstandes und somit $\mathrm{zu}$ einer $\mathrm{Zu}$ nahme der Sinkgeschwindigkeit kommt (s. Abb. 1). Eine Steigerung der Feststoffkonzentration führt $\mathrm{zu}$ einer erhöhten Clusterbildungswahrscheinlichkeit und zu größeren, gemeinsam sedimentierenden Komplexen. Die Größe der Cluster ist nach oben hin allerdings begrenzt, da deren Stabilität geringer wird [13]. Im Bereich höherer Feststoffkonzentrationen dominieren sedimentationsbehindernde Effekte. Zum einen nimmt die Suspensionsviskosität und -dichte mit der Feststoffkonzentration $\mathrm{zu}$, zum anderen erzeugt der Sedimentationsvorgang eine verstärkte Fluid-Gegenströmung, die die effektive Sinkgeschwindigkeit reduziert. Bei feindispersen Partikelsystemen wirkt auch die Brown'sche Molekularbewegung der Clusterbildung entgegen, ebenso wie elektrostatische Abstoßungskräfte. Durch interpartikuläre Anziehungskräfte wird die Clusterbildung jedoch verstärkt.

In polydispersen Suspensionen wirken grundsätzlich dieselben Mechanismen wie in Suspensionen mit monodispersen Partikeln. Jedoch kann man hier zusätzliche Segregationseffekte zwischen den unterschiedlichen Größenklassen beobachten. Im Falle einer stark verdünnten Suspension bewegen sich alle Partikel mit ihrer individuellen Stokes'schen Sinkgeschwindigkeit. Mit zunehmender Gesamtkonzentration tritt ein Mitnahmeeffekt auf, der kleinere Partikel aufgrund der hydrodynamischen Begünstigung durch größere Partikel mit erhöhter Geschwindigkeit sedi- 


\section{Ab der sog. Gel- punktkonzentration spricht man nicht mehr von einer Sus- pension, sondern von einem Sedi- ment, wobei haupt- sächlich mechani- sche Kräfte für des- sen Kompressions- verhalten verant- wortlich sind.}

mentieren lässt [12]. Auf der anderen Seite kann sich ein hoher Anteil großer Partikel durch den erzeugten Gegenstrom verdrängter Suspensionsflüssigkeit stark verlangsamend auf kleine Kornklassen auswirken. Im Extremfall führt der Fluidstrom zunächst zu einer Aufwärtsbewegung der kleinsten Partikel. Eine hohe Konzentration kleiner Partikel wiederum bewirkt ein langsameres Absinken der größeren Partikel durch die von ihnen verursachte Dichte- und Viskositätserhöhung der Suspension. Solange trotz gemeinsam sedimentierender Partikel eine breite Verteilung der Sinkgeschwindigkeiten vorliegt, spricht man von einer Schwarmsedimentation, die durch einen Klassiereffekt geprägt ist. Bei höheren Feststoffkonzentrationen, im Bereich der Zonensedimentation, sinken alle Partikel mit annähernd gleicher Geschwindigkeit ab. Beide Arten der Sedimentation sind von technischem Interesse. Für Klassieraufgaben eignet sich eine Schwarmsedimentation, da dabei Segregationseffekte zwischen den einzelnen Größenklassen einer Suspension auftreten. Beim Klären müssen auch feinste Partikel abgeschieden werden, wobei sich im Bereich der Zonensedimentation alle Partikel mit einheitlicher Sinkgeschwindigkeit absetzen [14].

Die Konzentration, bei der eine Schwarm- in eine Zonensedimentation übergeht, ist von den physikochemischen Suspensionseigenschaften oder auch einer wirksamen Zentrifugalbeschleunigung abhängig. Bei einer weiteren Erhöhung der Feststoffkonzentration bildet sich ab der sog. Gelpunktkonzentration [14] eine durch die gesamte Suspension gehende Struktur aus. Ab dieser Konzentration spricht man nicht mehr von einer Suspension, sondern von einem Sediment, wobei hauptsächlich mechanische Kräfte für dessen Kompressionsverhalten verantwortlich sind.

\subsection{Ansätze zur Berechnung der Sedimentation}

Die Basis für den halbempirischen Ansatz von Richardson und Zaki [15] bildet das von Kynch [16] formulierte Theorem, dass die Sinkgeschwindigkeit eines Partikels nur von der lokalen Partikelkonzentration in dessen unmittelbarer Umgebung abhängt:

$\frac{u_{\mathrm{p}}}{u_{\mathrm{St}}}=\left(1-c_{\mathrm{v}}\right)^{\mathrm{n}_{\mathrm{Ruz}}}$

Der Exponent dieser Gleichung ist eine Funktion der Reynoldszahl, wobei dieser für Reynoldszahlen unterhalb von $\operatorname{Re}_{\mathrm{p}}<0,8$ den Wert $n_{\text {Ruz }}=4,65$ besitzt. Der Ansatz ist auch für breit verteilte Partikelsysteme gültig. So- fern sich die Partikel während der Sedimentation nicht nennenswert behindern und sich somit gemäß ihrer individuellen, partikelgrößenabhängigen Sinkgeschwindigkeit absetzen, sedimentieren nur die gröbsten Fraktionen während der gesamten Sedimentationszeit in einer Umgebung, die der Ausgangskonzentration entspricht. Alle anderen Fraktionen setzen sich in einem Milieu ab, das nach und nach an den gröberen Fraktionen verarmt. Bürger et al. [17] berechnen auf numerischem Wege diese orts- und zeitabhängige Konzentrationsänderung für polydisperse Suspensionen aus kugelförmigen Partikeln.

Unter der Annahme, dass alle Partikel während der Absetzphase in einem Milieu sedimentieren, die der Anfangskonzentration entspricht, kann die Sinkgeschwindigkeit jeder Fraktion als Funktion der Anfangskonzentration nach Richardson und Zaki [15] berechnet werden. Scott [18] erweiterte den Ansatz von Richardson und Zaki für geflockte Systeme um einen sog. Flockenbildungsfaktor:

$k_{\mathrm{fl}}=\frac{c_{\mathrm{v}, \mathrm{fl}}}{c_{\mathrm{v}}}$

der das Verhältnis aus Flocken- $c_{\mathrm{v}, \mathrm{fl}} \mathrm{zu}$ Feststoffkonzentration $c_{\mathrm{v}}$ beschreibt. Die Flockenkonzentration ist eine scheinbare Konzentration, da in der Flocke immer Flüssigkeit mit eingeschlossen ist. Die Sinkgeschwindigkeit der Flocken ergibt sich dann zu:

$\frac{u_{\mathrm{fl}}}{u_{\mathrm{fl}, \mathrm{St}}}=\left(1-k_{\mathrm{fl}} c_{\mathrm{v}}\right)^{\mathrm{n}_{\mathrm{Ruz}}}$

Problematisch hierbei ist der Einfluss der Porosität. Bei Flocken mit einer relativ hohen Porosität wird der Absetzvorgang merklich durch die Durchströmung beeinflusst. Die Schwierigkeit besteht dann in der Kopplung von strukturanalytischen Untersuchungen mit Strömungsmodellen. Eine Zusammenfassung und kritische Bemerkungen dieser Ansätze sind in Tang und Raper [19] zu finden.

Weiterhin problematisch für eine Bestimmung der Sedimentationsgeschwindigkeit ist, dass weder die Flockendichte noch eine Flockengröße bekannt ist. Zur Bestimmung dieser Parameter stellte Freidinger [20] die Gleichung nach Scott um:

$u_{\mathrm{fl}}^{1 / 4,65}=-u_{\mathrm{fl}, \mathrm{St}}^{1 / 4,65} k_{\mathrm{fl}} c_{\mathrm{v}}+u_{\mathrm{fl}, \mathrm{St}}^{1 / 4,65}$

Trägt man nun die Sinkgeschwindigkeit der Flocken mit der Potenz nach Richardson und Zaki gegenüber der Feststoffkonzentration auf, so ergibt sich das sog. Freidinger-Diagramm [20].

Die Sinkgeschwindigkeit nimmt im Bereich der Zonensedimentation linear mit steigender 
Feststoffkonzentration ab. Aus dem extrapolierten Abszissenabschnitt kann der Flockenbildungsfaktor ermittelt werden. Die Extrapolation des Ordinatenwertes führt zur Sinkgeschwindigkeit der Flocken bei unendlicher Verdünnung. Aus den extrapolierten Werten der Stokes'schen Sinkgeschwindigkeit der Flocken und des Flockenbildungsfaktors errechnet sich dann der Äquivalentdurchmesser $x_{\mathrm{p}, \mathrm{fl}}$ und die Porosität $\varepsilon_{\mathrm{fl}}$ einer Kugel:

$x_{\mathrm{p}, \mathrm{fl}}=\sqrt{\frac{18 \eta k_{\mathrm{fl}} u_{\mathrm{fl}, \mathrm{St}}}{\left(\rho_{\mathrm{p}}-\rho_{\mathrm{l}}\right) C \mathrm{~g}}}$

$\varepsilon_{\mathrm{fl}}=1-\frac{1}{k_{\mathrm{fl}}}$

Die im Folgenden diskutierten Sedimentationsuntersuchungen wurden mit einem Titandioxid durchgeführt, bestehend aus sehr feinen, nahezu kugelförmigen Primärpartikeln im Bereich von etwa $300 \mathrm{~nm}$. Der isoelektrische Punkt befindet sich bei einem $\mathrm{pH}$-Wert von etwa 7. Sowohl bei einem $\mathrm{pH}$-Wert von ca. 3 als auch bei einem pH-Wert von 11 beträgt das Zetapotenzial ungefähr $50 \mathrm{mV}$. Die pH-Wert-Abhängigkeit des verwendeten Produktes ist nahezu punktsymmetrisch zum isoelektrischen Punkt. Die Versuche wurden an einer Manometerzentrifuge [14] durchgeführt.

\subsection{Einfluss des pH-Wertes}

Dargestellt ist in Abb. 2 die Sinkgeschwindigkeitsverteilung in Abhängigkeit des pH-Wertes. Bei einem $\mathrm{pH}$-Wert von 3 liegt die Suspension stabilisiert vor, was zu einer sehr breiten Sinkgeschwindigkeitsverteilung führt. Auch bei einem pH-Wert von 5 ist keine Änderung des Sedimentationsverhaltens $\mathrm{zu}$ erkennen. Die elektrostatische Abstoßung zwischen den Partikeln ist noch groß genug, um Koagulationseffekte zu verhindern. Am isoelektrischen Punkt kommt es jedoch zu einer signifikanten Erhöhung der Sinkgeschwindigkeit. Nahezu der gesamte Feststoff sedimentiert mit einer einheitlichen Sinkgeschwindigkeit in der Zonensedimentation. Die attraktiven interpartikulären Wechselwirkungen sind so stark, dass auch die gröberen Partikel in die Koagulate eingeschlossen werden. Eine Verschiebung des $\mathrm{pH}$-Wertes in den basischen Bereich hinein führt zu einer erneuten Stabilisierung der Suspension und $\mathrm{zu}$ einer Verbreiterung der Sinkgeschwindigkeitsverteilung. Obwohl die Zetapotenziale der Suspensionen bei $\mathrm{pH}=9$ und $\mathrm{pH}=11$ betragsmäßig gleich groß sind wie diejenigen bei $\mathrm{pH}=5$ bzw. $\mathrm{pH}=3$, sind die Sinkgeschwindigkeiten der Partikel im sauren Bereich etwas langsamer als im basischen.

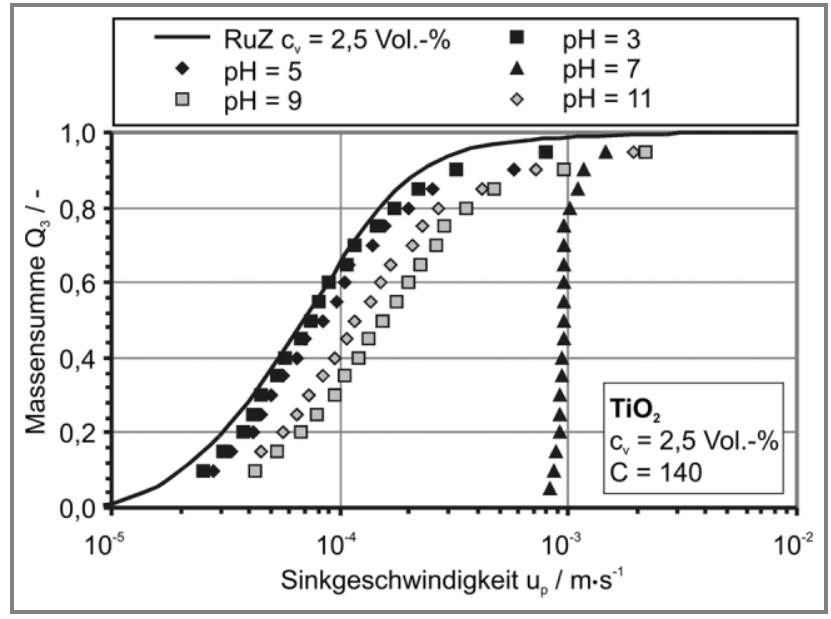

Abbildung 2. Einfluss des pH-Wertes auf das Sedimentationsverhalten von $\mathrm{TiO}_{2}$ (nach Beiser [14]).

Der wesentliche Unterschied liegt in der Belegung der Oberfläche. Während im sauren Bereich die Grenzfläche maßgeblich mit Protonen besetzt ist, befinden sich im basischen Bereich vorwiegend $\mathrm{OH}^{-}$-Ionen auf der Oberfläche. Dies könnte die unmittelbare Anordnung der ersten Wasserschichten beeinflussen, was zu einer unterschiedlich stark ausgeprägten Schicht strukturierten Wassers um die Partikel führen würde [14, 21].

\subsection{Einfluss der lonenstärke}

Hierfür ist zunächst eine Absenkung des $\mathrm{pH}$ Wertes durch Zugabe von Salpetersäure in den stabilisierten Bereich notwendig. Geringste Mengen einer Zugabe von $\mathrm{Na}_{2} \mathrm{SO}_{4}$ (Abb. 3) führen bereits $\mathrm{zu}$ einer Beschleunigung der Sedimentationskinetik. Geringe Salzmengen können ausreichen, um erste Koagulations-

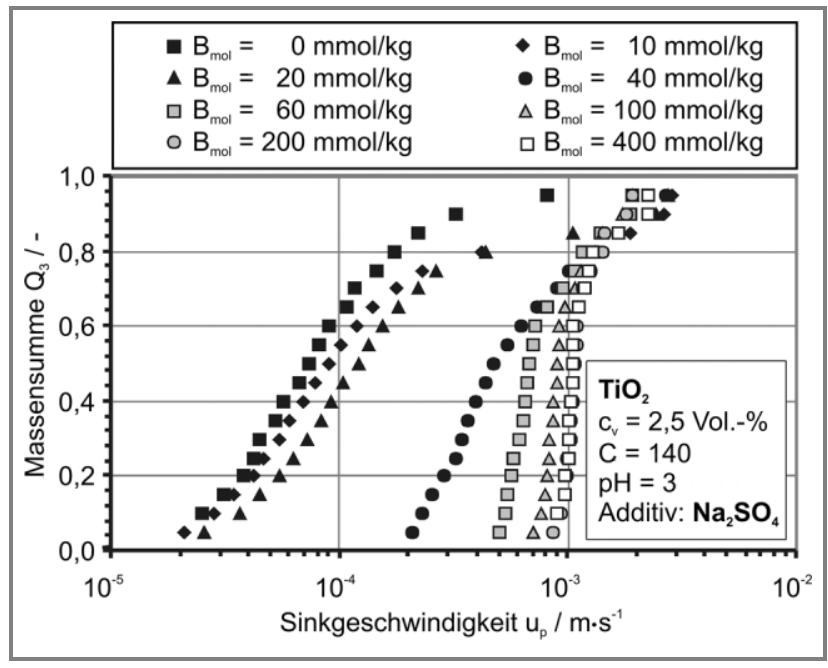

Abbildung 3. Einfluss einer $\mathrm{Na}_{2} \mathrm{SO}_{4}$ Zugabe auf das Sedimentationsverhalten (nach Beiser [14]). 
Die Sinkgeschwindigkeit gemeinsam sedimentierender Koagulate ist dabei mit derjenigen eines Partikelsystems am isoelektrischen Punkt vergleichbar. effekte hervorzurufen. Da das Zetapotenzial in diesem Bereich jedoch noch sehr hoch ist, könnte aber auch eine Beeinflussung der Dicke der Hydrathülle durch das Salz für das veränderte Sedimentationsverhalten verantwortlich sein. Obwohl der Einfluss von Hydratationskräften mit steigendem Elektrolytgehalt nicht auszuschließen ist, sind die Sinkgeschwindigkeitserhöhungen wohl mehr den Koagulationseffekten zuzuschreiben. Eine maximale Destabilisierung wird erst ab $200 \mathrm{mmol} / \mathrm{kg}$ erreicht. Die Sinkgeschwindigkeit der gemeinsam sedimentierenden Koagulate ist dabei mit derjenigen eines Partikelsystems am isoelektrischen Punkt vergleichbar. Lediglich die gröbsten Fraktionen zeigen eine etwas höhere Sinkgeschwindigkeit, was auf geringfügig schwächere Bindungskräfte innerhalb der Koagulate hindeutet. Versuche mit höherwertigen Ionen zeigen außerdem, dass diese hinsichtlich der Abschirmungswirkung nicht effektiver zu sein scheinen als die zweiwertigen Ionen.

Das elektrostatische Verhalten der Partikel ist nicht nur vom Salzgehalt abhängig, sondern auch von der Kinetik der Adsorption. Da sich eine Destabilisierung der Suspensionen in einem Beladungsbereich einstellt, in dem eine kleine Änderung der Beladung eine große Änderung der Grenzflächeneigenschaften hervorruft, kann eine kritische Salzmenge nur grob abgeschätzt werden. Es treten bei diesem Stoffsystem Koagulationseffekte auf, obwohl das Gesamtwechselwirkungspotenzial noch ein ausgeprägtes Maximum aufweist. Bei der jeweiligen kritischen Salzkonzentration tritt jedoch ein sekundäres Minimum im Bereich von etwa $-2,5 \mathrm{kT}$ auf. Somit findet hier eine Koagulation im sekundären Minimum statt.

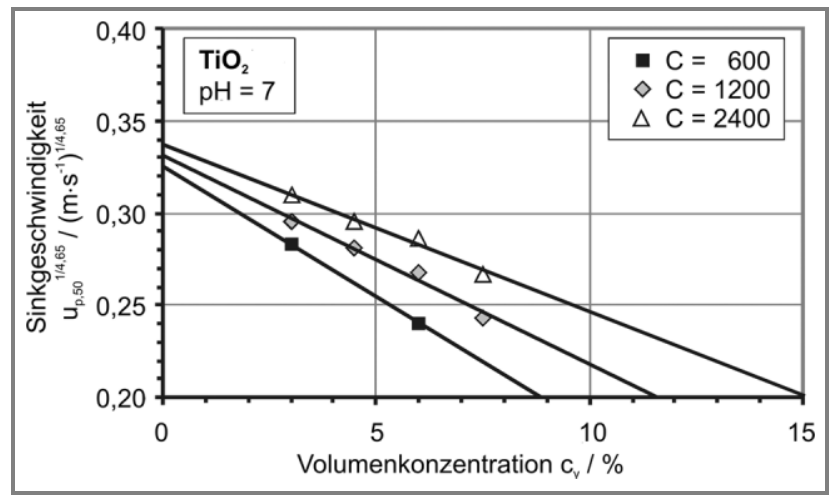

Abbildung 4. Einfluss der Zentrifugalbeschleunigung auf das Sedimentationsverhalten von $\mathrm{TiO}_{2}$ (nach Beiser [14]).

\subsection{Einfluss der Zentrifugal- beschleunigung}

Im sauren Bereich liegen die Partikel wiederum stabilisiert vor. In diesem Fall werden alle Verteilungen der auf den $C$-Wert bezogenen Sinkgeschwindigkeiten, unabhängig von der Zentrifugalbeschleunigung, durch eine einzige Kurve beschrieben. Eine Übertragung des Sedimentationsverhaltens von einer Zentrifugalbeschleunigung auf eine andere ist also für stabilisierte Suspensionen möglich. Bei destabilisierten Suspensionen sind nach Beiser [14] deutliche Abweichungen festzustellen. Bei höheren Zentrifugalbeschleunigungen weisen die Partikel eine geringere äquivalente Sinkgeschwindigkeit im Erdschwerefeld auf, was wiederum auf eine Koagulatzerstörung durch die gesteigerten Scherkräfte während des Absetzvorgangs zurückzuführen ist. Bei höheren Zentrifugalbeschleunigungen setzen sich somit immer kleinere Koagulate ab.

In Abb. 4 ist der Einfluss der Feststoffkonzentration auf das Absetzverhalten der gemeinsam sedimentierenden Koagulate bei unterschiedlichen Zentrifugalbeschleunigungen dargestellt. Bei größeren Zentrifugalbeschleunigungen ist der Gradient der Sinkgeschwindigkeitsabnahme $u_{\mathrm{p}, 50}{ }^{1 / 4,65}$ flacher. Die Koagulate behindern sich somit weniger, was auf eine abnehmende Flockenporosität hindeutet. Durch Extrapolation lassen sich aus der Freidinger-Auftragung die Stokes'sche Sinkgeschwindigkeit der Flocken und der Flockenbildungsfaktor bestimmen, aus denen wiederum die Flockenporosität und der Durchmesser einer Flocke berechnet werden kann, die ein äquivalentes Stokes'sches Sedimentationsverhalten aufweist wie die untersuchten Koagulate. Sowohl der äquivalente Stokes'sche Flockendurchmesser als auch -porosität nehmen mit steigender Zentrifugalbeschleunigung ab. Die Flocken werden demzufolge nicht nur von den stärkeren Scherkräften zerstört, sondern gleichzeitig auch kompaktiert. Durch die kompakteren Koagulate wird die Kontaktstellenzahl der Partikel innerhalb der Flocken erhöht, wodurch die Haltekräfte zunehmen. Es können immer größere Partikel in den Koagulaten gehalten werden.

\section{Physikochemische Einflüsse auf die Filtration}

In den folgenden Ausführungen sei schließlich eine kuchenbildende Filtration betrachtet. Sie ist dadurch charakterisiert, dass sich Feststoffpartikel zunächst an einem Filtermedium und anschließend an der Oberfläche eines sich 
bildenden Filterkuchens abscheiden. Die Suspensionsflüssigkeit strömt durch den Kuchen und das Filtermedium hindurch, wobei der Flüssigkeits- und der Feststoffstrom gleichgerichtet sind. Das treibende Potenzial für die Strömung der Flüssigkeit ist eine Druckdifferenz zwischen der Kuchenoberfläche und der Unterseite des Filtermediums. Die das Filtermedium verlassende, als feststofffrei angenommene Flüssigkeit wird als Filtrat bezeichnet.

\subsection{Ansätze zur Berechnung der Filtration}

Unter der Annahme zeitlich und örtlich konstanter Eigenschaften von Suspension und Kuchen, d.h. unter Ausschluss von Sedimentation und kompressiblem Verhalten des Filterkuchens, ergibt sich unter Voraussetzung einer konstanten Druckdifferenz $\Delta p$ durch Integration über der Zeit und Umformung eine Geradengleichung, die die Kinetik des Kuchenaufbaus beschreibt:

$\frac{t_{\mathrm{F}}}{V_{\mathrm{F}}}=C_{1} V_{\mathrm{F}}+C_{2}$

Im Faktor $C_{1}$ verbirgt sich der Widerstand des Filterkuchens, in der Konstanten $C_{2}$ der Filtermediumwiderstand. Filterkuchen, die aus Suspensionen feinstdisperser Partikel gewonnen werden, zeigen hingegen ein kompressibles Materialverhalten. Dabei sind die Porosität und der spezifische Widerstand Funktionen des auf den Feststoff wirkenden mechanischen Drucks $p_{\mathrm{s}}$. Die Partikel selbst müssen dabei nicht kompressibel sein. Einen umfassenden Überblick über die Ansätze zur Beschreibung von kompressiblen Prozessen und die Ursachen kompressiblen Materialverhaltens gibt Alles [22].

Kompressible Kuchen packen bei höheren Drücken dichter. Dabei müssen Kuchenschichten nahe dem Filtertuch die Masse und zusätzlich die Reibungskräfte der Strömung in darüberliegenden Schichten aufnehmen. Die mechanische Belastung im Kuchen und bei kompressiblem Verhalten auch die Porosität hängen vom Ort ab. Der hydraulische Widerstand ist wiederum eine sehr sensible Funktion der Porosität und damit im kompressiblen Fall ebenfalls stark ortsabhängig. Durch Überlagerung immer weiterer Kuchenschichten wachsen während des Kuchenaufbaus der Druckverlust des Fluids bis zur jeweils betrachteten Kuchenschicht und damit der lokale Feststoffgerüstdruck $p_{\mathrm{s}}$ stetig an. Dies führt zu einer fortschreitenden Nachkonsolidierung und zu einer Veränderung der Porosität bzw. des hydraulischen Widerstandes. Bei den sog. "super-compactible“ Filterkuchen bildet sich an der Filtermembran eine sog. „skin-Schicht“ aus, die durch einen sehr hohen Kuchenwiderstand charakterisiert ist und eine weitere Filtration nahezu unmöglich macht.

Tiller [23] beschreibt das kompressible Materialverhalten bei der Filtration, Kompression und Durchströmung durch eine Kombination von physikalischem Modell und empirischen Materialfunktionen. Vorausgesetzt werden ein Newton'sches Verhalten des Fluids, starre Partikel und vernachlässigbare Trägheitskräfte. Das physikalische Modellgleichungssystem zur Beschreibung der lokalen Zustände beinhaltet weiterhin ein Durchströmungsgesetz, das zusätzlich die Wanderungsgeschwindigkeit des Feststoffes berücksichtigt. Einbezogen werden die lokale Kräftebilanz und die Kontinuitätsgleichung. In dem System sind die Materialfunktionen $\varepsilon=f\left\{p_{\mathrm{s}}\right\}$ und $a=f\left(p_{\mathrm{s}}\right\}$ eingebettet, die den Einfluss der mechanischen Beanspruchung durch einaxiales Pressen auf die Materialparameter Porosität und Durchströmungswiderstand mathematisch beschreiben.

Tiller [23 - 25] gibt verschiedene geeignete Funktionen für Widerstand $a$ und Porosität $\varepsilon$ an. Unter anderem sind dies jedoch meistens Potenzansätze der folgenden Form:

$(1-\varepsilon)=\left(1-\varepsilon_{0}\right)\left(1+\frac{p_{\mathrm{s}}}{p_{\mathrm{s}, 0}}\right)^{\beta}$

und

$a=a_{0}\left(1+\frac{p_{\mathrm{s}}}{p_{\mathrm{s}, 0}}\right)^{\mathrm{n}}$

Neben der differentiellen Vorgehensweise existieren auch Ansätze, die die Kuchenbildung mit kompressiblen Kuchen unter Verwendung von Mittelwerten für Porosität und spezifischen Widerstand beschreiben [26]. Einen äquivalenten Weg, jedoch über eine direkte empirische Modifikation der Kuchenbildungsgleichung, schlägt dagegen Nicolaou [27] vor.

\subsection{Einfluss von Feststoffgerüstdruck und $\mathrm{pH}-$ Wert}

Bei industriellen Filtrationsprozessen ist man auf eine Mindestpermeabilität des entstehenden Filterkuchens angewiesen, um einen Kuchenaufbau in wirtschaftlich vertretbaren Zeiten zu realisieren. Der Kuchenwiderstand $a$ bzw. dessen Kehrwert, die Permeabilität $k$, sind somit entscheidende Materialparameter bei der Auswahl des Trennprinzips. Ist die Permeabilität gering, baut sich ein Filterkuchen
Bei industriellen Filtrationsprozessen ist man auf eine Mindestpermeabilität des entstehenden Filterkuchens angewiesen, um wirtschaftlich vertretbare Zeiten realisieren zu können. 


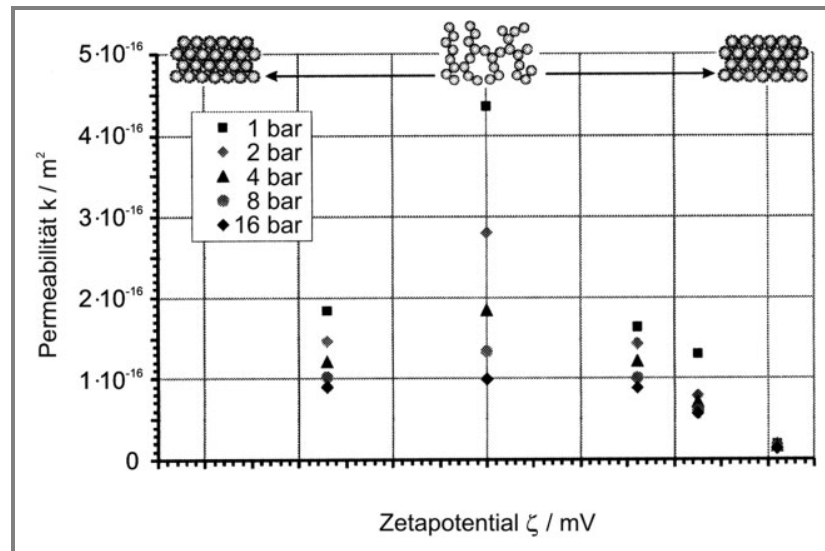

Abbildung 5. Permeabilität als Funktion des Zetapotenzials bei unterschiedlichen Gerüstdruckniveaus (nach Erk [28]).

nur langsam auf und der Flüssigkeitstransport durch Kuchen und Membran ist stark eingeschränkt. Eine Separation aufgrund zu geringer Permeabilität ist insbesondere für feinpartikuläre Suspensionen problematisch, da die Permeabilität näherungsweise proportional zum Quadrat der Partikelgröße ist [9]. Entscheidend sind jedoch für feinstpartikuläre oder nanoskalige Partikelsysteme die vorliegenden interpartikulären Kräfte bzw. die daraus resultierende Mikrostruktur des Haufwerks.

Abb. 5 zeigt den Durchströmungswiderstand als Funktion des Feststoffgerüstdrucks $p_{\mathrm{s}}$ für ein $\mathrm{Al}_{2} \mathrm{O}_{3} /$ Wasser-System bei unterschiedlichen, durch das Zetapotenzial charakterisierten Wechselwirkungszuständen. Mit steigendem Feststoffgerüstdruck kommt es zu einer Verdichtung der Packungsstruktur und somit zu einer Reduktion der Permeabilität. Bei hohen Werten des Zetapotenzials, d. h. bei abstoßenden interpartikulären Kräften ist der

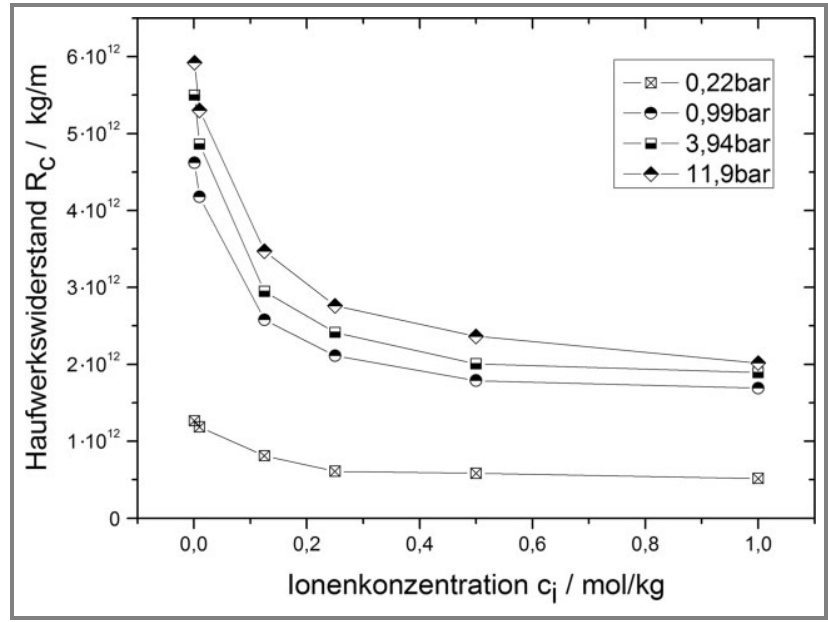

Abbildung 6. Einfluss des Feststoffgerüstdruckes und der lonenstärke auf den Haufwerkswiderstand.
Durchströmungswiderstand je nach Druckniveau um mehr als eine Größenordnung höher als am isoelektrischen Punkt. Bei dieser Auftragung der Permeabilität als Funktion des Zetapotenzials tritt die Symmetrie relativ zum isoelektrischen Punkt deutlich hervor. Schematisch ist in dieser Abbildung auch die zu erwartende Mikrostruktur eingezeichnet. Derartige Effekte wurden auch von anderen Autoren für unterschiedliche Stoffsysteme festgestellt [29-32].

\subsection{Einfluss der lonenstärke}

Die Zugabe eines Salzes verändert, ebenso wie der $\mathrm{pH}$-Wert, die Struktur der Doppelschichten in der näheren Umgebung der Partikel. Durch die Erhöhung der Konzentration des Elektrolyten in der Flüssigkeit resultiert eine Kompression der Schichten bzw. eine Reduktion der Debye-Länge. Der Durchströmungswiderstand nimmt nach Abb. 6 mit zunehmender Ionenstärke $a b$, was zunächst einmal auf einen strukturellen Effekt zurückzuführen ist. Mit zunehmender Ionenstärke wird die Überschussladung reduziert, wobei die Aggregationsneigung der Partikel dann zunimmt. Dies führt dazu, dass wiederum größere $\mathrm{Ag}$ gregate entstehen, die zu einer Porositätserhöhung und damit höherer Permeation führen. Derartige Ergebnisse sind in diesem Ausmaß immer dann zu beobachten, wenn die Struktur der Aggregate vor der Bildung des Filterkuchens generiert wird. In diesem Fall wird eine festgelegte Agglomeratstruktur filtriert, die durch die Wirkung des Feststoffgerüstdrucks strukturell nicht entscheidend verändert wird. Es ist aber auch denkbar, eine im Haufwerk fixierte Struktur einer Veränderung der Ionenstärke auszusetzen und dabei den Einfluss der Doppelschichtveränderung auf die Durchströmungseigenschaften $\mathrm{zu}$ bestimmen. Mit dieser Aufgabenstellung hat sich Schäfer [33] auseinandergesetzt und bei einer fixierten Struktur über eine $\mathrm{pH}$-Wertverschiebung festgestellt, dass eine Veränderung der Doppelschicht einen erheblichen Einfluss auf die Permeabilität hat.

\subsection{Strömungspotenzial und elektroosmotischer Fluss}

Die Wechselwirkung zwischen der Flüssigkeit und den Partikeln eines Haufwerks wird in erster Linie durch die hydrodynamischen Kräfte bestimmt. Bei feinsten oder nanoskaligen Partikelsystemen sind jedoch aufgrund der Wirkung der physikochemischen Wechsel- 
wirkung elektrokinetische Effekte zu berücksichtigen. Diese sind zunächst von den elektrostatischen Effekten zu unterscheiden, da über die Suspensionsvorbereitung die Agglomeratstruktur festgelegt ist. Elektrokinetische Effekte kommen dadurch zustande, dass die strömende Flüssigkeit Gegenionen aus dem diffusen Teil der Doppelschicht abschert, sodass es zu einem Ladungstransport, dem sog. Strömungsstrom $I_{\text {str }}$ kommt. Der Ladungstransport wird durch einen Gegenstrom ausgeglichen, der auf einer Ionenwanderung entgegen der Strömung und auf einer Oberflächenleitfähigkeit beruht. Das treibende Potenzial für diesen Gegenstrom ist als Strömungspotenzial $U_{\text {str }}$ zwischen beiden Seiten des Haufwerks messbar:

$U_{\text {str }}=\frac{\varepsilon_{0} \varepsilon_{\mathrm{r}} \zeta \Delta p}{\eta\left(K_{\mathrm{L}}+2 K_{\sigma} / r_{a}\right)}$

Das Strömungspotenzial wurde von Schäfer [34] bei einer druckgetriebenen Durchströmung eines Haufwerks mit Elektrolytlösungen bei verschiedenen $\mathrm{pH}$-Werten ermittelt. Die Ionenstärke und damit die Debye-Länge wurden dabei konstant gehalten.

Für jede verwendete Elektrolytlösung ist das Strömungspotenzial abhängig und direkt proportional zur angelegten Druckdifferenz. Bei hohen $\mathrm{pH}$-Werten, wobei bei den untersuchten Titandioxidpartikeln ein negatives Zetapotenzial vorlag, ergab sich ein negatives Strömungspotenzial. Im sauren und im neutralen Bereich ist dagegen ein positives Potenzial als Folge eines positiven Zetapotenzials messbar. Es zeigten sich auch Abweichungen von dem in der Suspension gemessenen, monotonen Verlauf des Zetapotenzials. Da das Zetapotenzial jedoch konzentrationsabhängig ist, kann man im Haufwerk nicht den gleichen Verlauf wie in der Suspension erwarten.

Umgekehrt kann somit die Strömung durch ein partikuläres Haufwerk nicht nur als Folge der Ausbildung eines Strömungspotenzials durch ein Gravitations- bzw. Zentrifugalfeld oder eines Druckgradienten getrieben werden. Mit Hilfe von elektrischen Feldern ist ebenfalls ein Flüssigkeitsstrom durch die Haufwerksstruktur zu erzielen. Dabei werden durch ein angelegtes elektrisches Potenzial die beweglichen Ionen im diffusen Teil der elektrochemischen Doppelschicht beschleunigt. Die Ionen bewegen das sie umgebende Wasser aufgrund der viskosen Widerstandskraft durch die Poren. Dieser elektroosmotische Fluss ist mit:

$\dot{V}_{\mathrm{eo}}=-\frac{\varepsilon_{0} \varepsilon_{\mathrm{r}} \zeta I}{\eta K_{\mathrm{L}}}$

eine Funktion der elektrischen Stromstärke $I$. Der elektroosmotische Volumenstrom erhöht

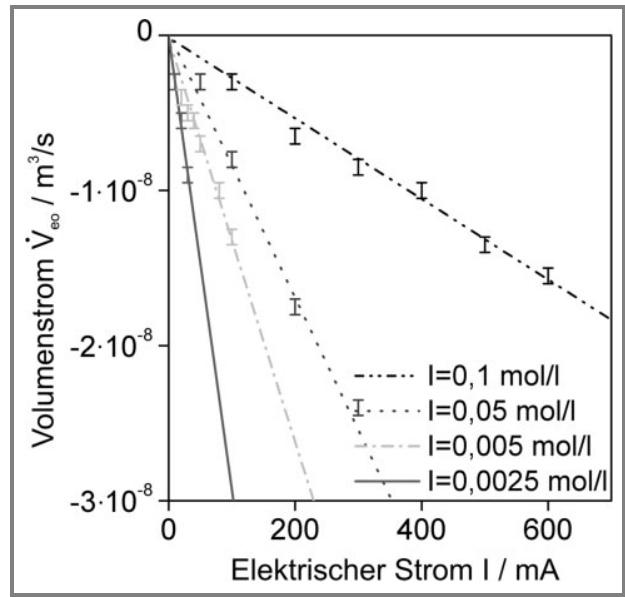

oder vermindert die druckgetriebene Durchströmung und kann auch als eine „elektroosmotische Pumpe“ betrieben werden. Der gesamte Volumenstrom ergibt sich als Summe der hydraulisch und elektroosmotisch getriebenen Durchströmung. Abb. 7 zeigt beispielhaft den elektroosmotischen Volumenstrom als Funktion des angelegten Stroms bei einem konstanten $\mathrm{pH}$-Wert und veränderten Ionenstärken.

Bei dem untersuchten $\mathrm{pH}$-Wert von 7 liegt ein positives Zetapotenzial vor. Dieses bewirkt eine negative Fließrate, die bei hoher Ionenstärke wesentlich kleiner ist als bei niedriger Ionenstärke. Bei niedriger Ionenstärke ist die Ausdehnung der elektrostatischen Doppelschicht groß, in denen die Gegenionen im Überschuss vorliegen. Durch ein angelegtes elektrisches Feld werden nun die Gegenionen beschleunigt, wobei diese das sie umgebende Wasser mitbewegen und so ein Volumenstrom entsteht. Bei hoher Ionenstärke ist dagegen die Doppelschicht entsprechend komprimiert. Somit stehen für den elektroosmotischen Transport weniger Ionen zur Verfügung. Außerhalb der Doppelschicht ist hingegen die Anzahl der Anionen und Kationen gleich, was $\mathrm{zu}$ einer Beschleunigung der Ionen in beide Richtungen führt.

\section{Zusammenfassung}

In dem vorliegenden Beitrag wird dargestellt, dass die physikochemischen Bedingungen für das Separationsverhalten von feinsten bzw. nanoskaligen Partikeln mittels mechanischer Trennverfahren entscheidend sind. Wesentliche Einflussgrößen sind der pH-Wert und die Ionenstärke bzw. alle Verfahren, die den elektrischen Ladungszustand der Partikelgrenzfläche verändern. Dadurch wird zunächst die Struktur der gebildeten Agglomerate beein-
Abbildung 7. Einfluss des Stroms und der lonenstärke auf den elektroosmotischen Volumenstrom (nach Schäfer [34]).
Für jede verwendete Elektrolytlösung ist das Strömungspotenzial abhängig und direkt proportional zur angelegten Druckdifferenz. 
flusst, aber auch die Ausdehnung der Doppelschicht. Eine gezielte Kontrolle dieser Einflussgrößen ist damit Voraussetzung für eine sichere Separation in praktischen Anwendungsfällen.

\section{Prof. Dr. H. Nirschl}

(hermann.nirschl@mvm.uni-karlsruhe.de), Institut für Mechanische Verfahrenstechnik, Universität Karlsruhe (TH),

D-76131 Karlsruhe, Germany.

\section{Formelzeichen}

\section{Lateinische Buchstaben}

\begin{tabular}{|c|c|c|}
\hline$A$ & {$[\mathrm{~J}]$} & $\begin{array}{l}\text { Hamaker-Konstante in } \\
\text { wässrigem Medium }\end{array}$ \\
\hline C & {$[-]$} & $\begin{array}{l}\text { Vielfaches der Erdbeschleu- } \\
\text { nigung, } C \text {-Wert }\end{array}$ \\
\hline$c_{\mathrm{v}}$ & {$[-]$} & $\begin{array}{l}\text { Feststoffvolumenkonzentra- } \\
\text { tion }\end{array}$ \\
\hline$c_{\mathrm{v}, \mathrm{fl}}$ & {$[-]$} & $\begin{array}{l}\text { Flockenvolumenkonzentra- } \\
\text { tion }\end{array}$ \\
\hline$F_{\mathrm{r}}$ & {$[\mathrm{N}]$} & repulsive Kraft \\
\hline$g$ & {$\left[\mathrm{~m} / \mathrm{s}^{2}\right]$} & Gravitationskonstante \\
\hline$H$ & {$[\mathrm{~m}]$} & Partikelabstand \\
\hline$I$ & {$[\mathrm{~mA}]$} & elektrischer Strom \\
\hline$I_{\mathrm{Str}}$ & {$[\mathrm{mA}]$} & Strömungsstrom \\
\hline$k$ & {$\left[1 / \mathrm{m}^{2}\right]$} & Permeabilität \\
\hline$k_{\mathrm{fl}}$ & {$[-]$} & Flockenbildungsfaktor \\
\hline$K_{\mathrm{L}}$ & {$[\mathrm{mS} / \mathrm{m}]$} & $\begin{array}{l}\text { Leitfähigkeit der Elektro- } \\
\text { lytlösungen }\end{array}$ \\
\hline$K_{\sigma}$ & {$[\mathrm{S}]$} & $\begin{array}{l}\text { Leitfähigkeit der Partikel- } \\
\text { oberfläche }\end{array}$ \\
\hline$L$ & {$[\mathrm{~m}]$} & geometrische Konstante \\
\hline$n$ & {$[-]$} & Anpassungsexponent \\
\hline$n_{\mathrm{RuZ}}$ & {$[-]$} & $\begin{array}{l}\text { Exponent nach Richardson } \\
\text { und Zaki }\end{array}$ \\
\hline$p_{\mathrm{s}}$ & {$[\mathrm{Pa}]$} & $\begin{array}{l}\text { Feststoffgerüstdruck (mit } \\
\text { Index „0“: Bezugszustand) }\end{array}$ \\
\hline$\Delta p$ & {$[\mathrm{~Pa}]$} & treibendes Druckgefälle \\
\hline
\end{tabular}

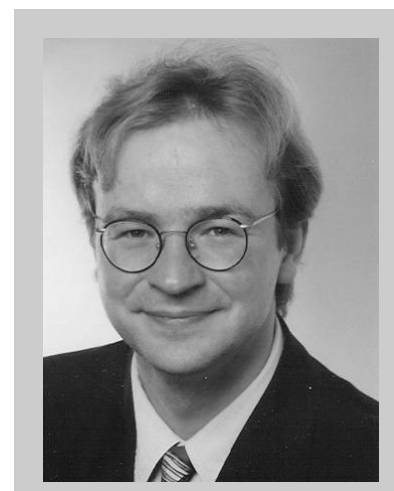

Hermann Nirschl, geb. 1964, studierte von 1984 bis 1989 an der Technischen Universität in München Technologie und Biotechnologie. Mit einem Forschungsstipendium ging er für ein Jahr an die University of California Davis (USA). Bis zu seiner Habilitation 1997 war er an der TU München wissenschaftlich tätig. Von 1997 bis 2002 bekleidete er eine leitende Position bei der ESPE Dental AG Seefeld. Seit 2002 lehrt er an der Universität in Karlsruhe. Nirschl ist berufenes Mitglied in den GVC Fachausschüssen „Mechanische Flüssigkeitsabtrennung“ und „Grenzflächen in der Feststoffverfahrenstechnik“.

\begin{tabular}{|c|c|c|}
\hline$R_{\mathrm{c}}$ & {$[\mathrm{kg} / \mathrm{m}]$} & Haufwerkswiderstand \\
\hline $\operatorname{Re}_{\mathrm{p}}$ & {$[-]$} & $\begin{array}{l}\text { Reynoldszahl bezogen auf } \\
\text { den Partikeldurchmesser }\end{array}$ \\
\hline$r_{a}$ & {$[\mathrm{~m}]$} & Kurvenradius eines Partikels \\
\hline$t_{\mathrm{F}}$ & {$[\mathrm{s}]$} & Filtrationszeit \\
\hline$u_{\mathrm{fl}}$ & {$[\mathrm{m} / \mathrm{s}]$} & $\begin{array}{l}\text { Sinkgeschwindigkeit einer } \\
\text { Flocke }\end{array}$ \\
\hline$u_{\mathrm{fl}, \mathrm{St}}$ & {$[\mathrm{m} / \mathrm{s}]$} & $\begin{array}{l}\text { Stokes'sche Sinkgeschwin- } \\
\text { digkeit einer Flocke }\end{array}$ \\
\hline$u_{\mathrm{p}}$ & {$[\mathrm{m} / \mathrm{s}]$} & $\begin{array}{l}\text { Sinkgeschwindigkeit eines } \\
\text { Partikels }\end{array}$ \\
\hline$u_{\mathrm{St}}$ & {$[\mathrm{m} / \mathrm{s}]$} & $\begin{array}{l}\text { Stokes'sche Sinkgeschwin- } \\
\text { digkeit eines Partikels }\end{array}$ \\
\hline$U_{\text {Str }}$ & {$[\mathrm{mV}]$} & Strömungspotenzial \\
\hline$V$ & {$[J]$} & $\begin{array}{l}\text { Wechselwirkungspotenzial } \\
\text { (Indices: a - attraktiv; } \\
\text { r - repulsiv) }\end{array}$ \\
\hline$V_{\mathrm{F}}$ & {$\left[\mathrm{m}^{3}\right]$} & Filtratvolumen \\
\hline$\dot{V}_{\mathrm{eo}}$ & {$\left[\mathrm{m}^{3} / \mathrm{s}\right]$} & $\begin{array}{l}\text { elektroosmotischer Volu- } \\
\text { menstrom }\end{array}$ \\
\hline$x_{\mathrm{p}}$ & {$[\mathrm{m}]$} & Partikeldurchmesser \\
\hline$x_{\mathrm{p}, \mathrm{fl}}$ & {$[\mathrm{m}]$} & Flockendurchmesser \\
\hline
\end{tabular}

\section{Griechische Buchstaben}

\begin{tabular}{|c|c|c|}
\hline$a$ & {$[-]$} & $\begin{array}{l}\text { flächenbeladungsspezifi- } \\
\text { scher Durchströmungs- } \\
\text { widerstand (mit Index „0“: } \\
\text { Bezugszustand) }\end{array}$ \\
\hline$\beta$ & {$[-]$} & Anpassungsexponent \\
\hline$\varepsilon$ & {$[-]$} & $\begin{array}{l}\text { Porosität (mit Index „0“: } \\
\text { Bezugszustand) }\end{array}$ \\
\hline$\varepsilon_{0}$ & {$[-]$} & elektrische Feldkonstante \\
\hline$\varepsilon_{\mathrm{r}}$ & {$[-]$} & $\begin{array}{l}\text { relative Dielektrizitätskons- } \\
\text { tante }\end{array}$ \\
\hline$\varepsilon_{\mathrm{fl}}$ & {$[-]$} & Flockenporosität \\
\hline & [Pas ] & dynamische Viskosität \\
\hline & {$\left[\mathrm{m}^{-1}\right]$} & Debye-Hückel-Parameter \\
\hline$\rho_{1}$ & {$\left[\mathrm{~kg} / \mathrm{m}^{-3}\right]$} & Partikeldichte \\
\hline & {$\left[\mathrm{kg} / \mathrm{m}^{-3}\right]$} & Dichte der Flüssigkeit \\
\hline & [mV ] & Zetapotenzial \\
\hline
\end{tabular}

\section{Literatur}

[1] G. Lagaly, O. Schulz, R. Zimehl, Dispersionen und Emulsionen, Steinkopff-Verlag, Darmstadt 1997.

[2] P. W. Atkins, Physikalische Chemie, 2.Auflage, VCH, Weinheim 1996.

[3] H. C. Hamaker, Physica 1937, 4, 1058.

[4] E. Verwey, J. Overbeek, Theory of Stability of Lyophobic Colloids, Elsevier-Verlag, Amsterdam 1948.

[5] J. T. Overbeek, Pure Appl. Chem. 1980, 52, 1151.

[6] J. Lyklema, Fundamentals of Colloid and Interface Science, I und II, Acad. Press, London 1991.

[7] R. Hogg, T. Healy, D. Fuerstenau., Trans. Faraday Soc. 1966, 62, 1638.

[8] B. Derjaguin, L. A. Landau, Acta Phys. Chim. USSR 1941, 14, 633. 
[9] J. Heuser, Dissertation, Universität Karlsruhe (TH) 2003.

[10] R. J. Hunter, Zeta-Potential in Colloid Science, Acad. Press, London 1981.

[11] G. G. Stokes, Trans. Cambridge Phil. Soc. 1850, 9.

[12] G. Bickert, Dissertation, Universität Karlsruhe (TH) 1997.

[13] B. Koglin, Habilitation, Universität Karlsruhe (TH) 1971.

[14] M. Beiser, Dissertation, Universität Karlsruhe (TH) 2005.

[15] J. F. Richardson, W. N. Zaki, Trans. Inst. Chem. Eng. 1954, 32, 35.

[16] G. J. Kynch, Trans. Faraday Soc. 1952, 48, 166.

[17] R. Bürger, F. Concha, K.-K. Fjelde, K. H. Karlsen, Numerical Simulation of the Settling of Polydisperse Suspensions of Spheres, SFB 404, Mehrfeldprobleme in der Kontinuumsmechanik, Bericht 1999/02, Universität Stuttgart 1999.

[18] K. J. Scott, Trans. Inst. Min. Metall. 1968, 77, C85.

[19] P. Tang, J. A. Raper, Powder Technol. 2002, $123(2-3), 114$.

[20] R. Freidinger, Dissertation, Universität Stuttgart 1985.

[21] T. Sobisch et al., Filtration 2006, 6 (4), 313.
[22] C. Alles, Dissertation, Universität Karlsruhe 2000.

[23] F. M. Tiller, C. Yeh, W. F. Leu, Sep. Sci. Technol. 1987, 22, 1037.

[24] F. M. Tiller, W. P. Li, J. B. Lee, Water Sci. Technol.: Water Supply 2001, 10, 171.

[25] F. M. Tiller, J. H. Kwon, AIChE J. 1998, 10, 1259.

[26] H. Anlauf, FQSS Filtr. Sep. 1994, 8 (2), 63.

[27] J. Nicolaou, Habilitation, Universität Karlsruhe (TH) 1996.

[28] A. Erk, Dissertation, Universität Karlsruhe (TH) 2006.

[29] E. Iritani, Y. Toyoda, T. Murase, J. Chem. Eng. Jpn. 1997, 30, 614.

[30] R. Nabi et al., in 5thWorld Congress on Particle Technology, Orlando, Florida, April 2006.

[31] R. J. Wakeman, S. T. Thuraisingham, E. S. Tarleton, F\&S Filtr. Sep. 1989, 4, 277.

[32] K. Ohmori, C. E. Glatz, J. Membr. Sci. 1999 , 153, 23.

[33] B. Schäfer, H. Nirschl, Chem. Eng. Techn. 2005, 28 (8), 862. DOI: $10.1002 /$ ceat.200500079

[34] B. Schäfer, H. Nirschl, Chem. Ing. Tech. 2007, 79, (9), 1412. DOI: 10.1002/cite.200750110 